\title{
On the Alon-Tarsi Number and Chromatic-Choosability of Cartesian Products of Graphs
}

\author{
Hemanshu Kaul \\ Department of Applied Mathematics \\ Illinois Institute of Technology \\ Chicago, IL, U.S.A. \\ kaul@iit.edu
}

\author{
Jeffrey A. Mudrock \\ Department of Mathematics \\ College of Lake County \\ Grayslake, IL, U.S.A. \\ jmudrock@clcillinois.edu
}

Submitted: Mar 22, 2018; Accepted: Dec 12, 2018; Published: Jan 11, 2019

(C) The authors. Released under the CC BY-ND license (International 4.0).

\begin{abstract}
We study the list chromatic number of Cartesian products of graphs through the Alon-Tarsi number as defined by Jensen and Toft (1995) in their seminal book on graph coloring problems. The Alon-Tarsi number of $G, A T(G)$, is the smallest $k$ for which there is an orientation, $D$, of $G$ with max indegree $k-1$ such that the number of even and odd circulations contained in $D$ are different. It is known that $\chi(G) \leqslant \chi_{\ell}(G) \leqslant \chi_{p}(G) \leqslant A T(G)$, where $\chi(G)$ is the chromatic number, $\chi_{\ell}(G)$ is the list chromatic number, and $\chi_{p}(G)$ is the paint number of $G$. In this paper we find families of graphs $G$ and $H$ such that $\chi(G \square H)=A T(G \square H)$, reducing this sequence of inequalities to equality.

We show that the Alon-Tarsi number of the Cartesian product of an odd cycle and a path is always equal to 3 . This result is then extended to show that if $G$ is an odd cycle or a complete graph and $H$ is a graph on at least two vertices containing the Hamilton path $w_{1}, w_{2}, \ldots, w_{n}$ such that for each $i, w_{i}$ has a most $k$ neighbors among $w_{1}, w_{2}, \ldots, w_{i-1}$, then $A T(G \square H) \leqslant \Delta(G)+k$ where $\Delta(G)$ is the maximum degree of $G$. We discuss other extensions for $G \square H$, where $G$ is such that $V(G)$ can be partitioned into odd cycles and complete graphs, and $H$ is a graph containing a Hamiltonian path. We apply these bounds to get chromaticchoosable Cartesian products, in fact we show that these families of graphs have $\chi(G)=A T(G)$, improving previously known bounds.
\end{abstract}

Mathematics Subject Classifications: 05C30, 05C15

\section{Introduction}

In this paper all graphs are finite, and all graphs are either simple graphs or simple directed graphs. Generally speaking we follow West [23] for terminology and notation. 
List coloring is a well known variation on the classic vertex coloring problem, and it was introduced independently by Vizing [22] and Erdős, Rubin, and Taylor [6] in the 1970's. In the classic vertex coloring problem we wish to color the vertices of a graph $G$ with as few colors as possible so that adjacent vertices receive different colors, a so-called proper coloring. The chromatic number of a graph, denoted $\chi(G)$, is the smallest $k$ such that $G$ has a proper coloring that uses $k$ colors. For list coloring, we associate a list assignment, $L$, with a graph $G$ such that each vertex $v \in V(G)$ is assigned a list of colors $L(v)$ (we say $L$ is a list assignment for $G$ ). The graph $G$ is $L$-colorable if there exists a proper coloring $f$ of $G$ such that $f(v) \in L(v)$ for each $v \in V(G)$ (we refer to $f$ as a proper $L$-coloring for $G)$. The list chromatic number of a graph $G$, denoted $\chi_{\ell}(G)$, is the smallest $k$ such that $G$ is $L$-colorable whenever the list assignment $L$ satisfies $|L(v)| \geqslant k$ for each $v \in V(G)$. It is immediately obvious that for any graph $G, \chi(G) \leqslant \chi_{\ell}(G)$. Erdös, Taylor, and Rubin observed in [6] that bipartite graphs can have arbitrarily large list chromatic number. This means that the gap between $\chi(G)$ and $\chi_{\ell}(G)$ can be arbitrarily large, and we can not hope to find an upper bound for $\chi_{\ell}(G)$ in terms of just $\chi(G)$.

Graphs in which $\chi(G)=\chi_{\ell}(G)$ are known as chromatic-choosable graphs (see [17]). Many classes of graphs have been conjectured to be chromatic-choosable. The most well known conjecture along these lines is the List Coloring Conjecture (see [9]) which states that every line graph of a loopless multigraph is chromatic-choosable. In addition, total graphs ([3]) and claw free graphs ([8]) are conjectured to be chromatic-choosable. On the other hand, there are classes of graphs that are known to be chromatic-choosable. In 1995, Galvin [7] showed that the List Coloring Conjecture holds for line graphs of bipartite multigraphs, and in 1996, Kahn [13] proved an asymptotic version of the conjecture. Tuza and Voigt [21] showed that chordal graphs are chromatic-choosable, and Prowse and Woodall [18] showed that powers of cycles are chromatic-choosable. Recently, Noel, Reed, and $\mathrm{Wu}[16]$ proved Ohba's conjecture which states that every graph, $G$, on at most $2 \chi(G)+1$ vertices is chromatic-choosable.

In this paper, we continue this investigation of chromatic-choosability in the realm of Cartesian products of graphs. We study the list chromatic number of Cartesian products of graphs through the Alon-Tarsi number as defined by Jensen and Toft in their seminal 1995 book on graph coloring problems [12]. The Alon-Tarsi number (AT-number for short) of $G, A T(G)$, is the smallest $k$ for which there is an orientation, $D$, of $G$ with max indegree $k-1$ such that the number of even and odd circulations contained in $D$ are different. It follows from the Alon-Tarsi Theorem [2] that $\chi(G) \leqslant \chi_{\ell}(G) \leqslant A T(G)$. We are interested in finding $G$ for which these three parameters are equal.

In the next two subsections, we discuss the known bounds for the list chromatic number of the Cartesian product of graphs as well as the main tool we use to obtain our results: the Alon-Tarsi Theorem. In Section 2, we give a series of sharp bounds on the AT-number of Cartesian Products of the form $G \square H$, where $G$ is such that $V(G)$ can be partitioned into odd cycles and complete graphs, and $H$ is a traceable graph (a graph containing a Hamiltonian path). In Section 3, we apply the bounds from the previous section to give some examples of chromatic-choosable Cartesian products of graphs, and even more strongly we show that these graphs have $\chi(G)=\chi_{\ell}(G)=A T(G)$. 


\subsection{Cartesian Product of Graphs}

The Cartesian product of graphs $G$ and $H$, denoted $G \square H$, is the graph with vertex set $V(G) \times V(H)$ and edges created so that $(u, v)$ is adjacent to $\left(u^{\prime}, v^{\prime}\right)$ if and only if either $u=u^{\prime}$ and $v v^{\prime} \in E(H)$ or $v=v^{\prime}$ and $u u^{\prime} \in E(G)$. Note that $G \square H$ contains $|V(G)|$ copies of $H$ and $|V(H)|$ copies of $G$. It is also easy to show that $\chi(G \square H)=\max \{\chi(G), \chi(H)\}$. So, we have that $\max \{\chi(G), \chi(H)\} \leqslant \chi_{\ell}(G \square H)$.

There are few results in the literature regarding the list chromatic number of the Cartesian product of graphs. In 2006, Borowiecki, Jendrol, Král, and Miškuf [4] showed the following.

Theorem $1([4]) \cdot \chi_{\ell}(G \square H) \leqslant \min \left\{\chi_{\ell}(G)+\operatorname{col}(H), \operatorname{col}(G)+\chi_{\ell}(H)\right\}-1$.

Here $\operatorname{col}(G)$, the coloring number of a graph $G$, is the smallest integer $d$ for which there exists an ordering, $v_{1}, v_{2}, \ldots, v_{n}$, of the elements in $V(G)$ such that each vertex $v_{i}$ has at most $d-1$ neighbors among $v_{1}, v_{2}, \ldots, v_{i-1}$. The coloring number is a classic greedy upper bound on the list chromatic number, and it immediately implies that $\Delta(G)+1$ is an upper bound on the list chromatic number where $\Delta(G)$ is the maximum degree of $G$. Vizing [22] extended this by proving the list coloring version of Brooks' Theorem.

Theorem 2 ([22]). Suppose that $G$ is a connected graph with maximum degree $\Delta(G)$. If $G$ is neither a complete graph nor an odd cycle, then $\chi_{\ell}(G) \leqslant \Delta(G)$.

Borowiecki et al. [4] construct examples where the upper bound in their theorem is tight. Specifically, they show that if $k \in \mathbb{N}$ and $G$ is a copy of the complete bipartite

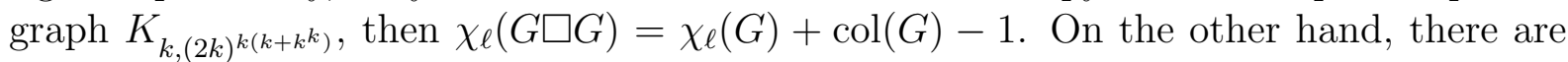
examples where the upper bounds from Theorems 1 and 2 are not tight. For example, suppose that $G$ is a copy of $C_{2 k+1} \square P_{n}$ where $n \geqslant 3$. Since $\chi_{\ell}\left(C_{2 k+1}\right)=\operatorname{col}\left(C_{2 k+1}\right)=3$ and $\chi_{\ell}\left(P_{n}\right)=\operatorname{col}\left(P_{n}\right)=2$, Theorem 1 tells us that $\chi_{\ell}(G) \leqslant 4$. Similarly, Theorem 2 tells us $\chi_{\ell}(G) \leqslant 4$, yet we will show below that $\chi_{\ell}(G)=3$. Another, more dramatic, example where Theorems 1 and 2 do not produce tight bounds is when we are working with the Cartesian product of two complete graphs. Suppose $m \geqslant n \geqslant 2$, and note that $K_{m} \square K_{n}$ is the line graph for the complete bipartite graph $K_{m, n}$. So, by Galvin's celebrated result ([7]): $m=\chi\left(K_{m} \square K_{n}\right)=\chi_{\ell}\left(K_{m} \square K_{n}\right)$. However, Theorem 1 only yields an upper bound of $\chi_{\ell}\left(K_{m} \square K_{n}\right) \leqslant m+n-1$ since $\chi_{\ell}\left(K_{m}\right)=\operatorname{col}\left(K_{m}\right)=m$. Similarly, Theorem 2 only tells us $\chi_{\ell}\left(K_{m} \square K_{n}\right) \leqslant m+n-2$.

Alon [1] showed that for any graph $G, \operatorname{col}(G) \leqslant 2^{O\left(\chi_{\ell}(G)\right)}$. Combining this result with Theorem 1 implies that we have an upper bound on $\chi_{\ell}(G \square H)$ in terms of only the list chromatic numbers of the factors. Borowiecki et al. [4] conjecture that a much stronger bound holds: there is a constant $A$ such that $\chi_{\ell}(G \square H) \leqslant A\left(\chi_{\ell}(G)+\chi_{\ell}(H)\right)$. While we will not address this conjecture in this paper, we will present results that are improvements on Theorem 1 when the factors in the Cartesian product satisfy certain properties. Our main aim in this note is to illustrate how to utilize the classic Alon-Tarsi Theorem in these situations to get better bounds on the AT-number and consequently the list chromatic number. 


\subsection{Alon-Tarsi Number}

Suppose graph $D$ is a simple digraph. We say that $E$ is a circulation contained in $D$ if $E$ is a spanning subgraph of $D$ and for each $v \in V(D), d_{E}^{-}(v)=d_{E}^{+}(v)$ (Note: $d_{E}^{-}(v)$ represents the indegree of $v$ in $E$ and $d_{E}^{+}(v)$ represents the outdegree of $v$ in $E$ ). We say a circulation is even (resp. odd) if it has an even (resp. odd) number of edges. Classic results in graph theory tell us that a circulation is a digraph which consists of Eulerian components. This means a circulation can be decomposed into directed cycles. Alon and Tarsi [2] use algebraic methods, subsequently called the Combinatorial Nullstellensatz, to obtain a remarkable relationship between a special orientation of a graph and a certain graph polynomial. The result is the celebrated Alon-Tarsi Theorem.

Theorem 3 (Alon-Tarsi Theorem). Let $D$ be an orientation of the simple graph $G$. Suppose that $L$ is a list assignment for $G$ such that $|L(v)| \geqslant d_{D}^{+}(v)+1$. If the number of even and odd circulations contained in $D$ differ, then there is a proper L-coloring for $G$. In addition, if the maximum indegree of $D$ is $m$ and the number of even and odd circulations contained in $D$ differ, then $\chi_{\ell}(G) \leqslant m+1$.

Recently, further implications of the Alon-Tarsi Theorem have appeared in the literature. Before mentioning one of these implications we need some terminology. For a graph $G$ suppose that for each $v \in V(G), k$ tokens are available at $v$. Two players called the marker and remover then play the following game on the graph $G$. For each round, the marker marks a non-empty subset, $M$, of vertices on the graph which uses one token for each marked vertex. The remover then selects a subset of vertices, $I \subseteq M$, to remove such that $I$ is an independent set of vertices in $G$. The marker wins by marking a vertex that has no tokens, and the remover wins by removing all the vertices from the graph. A graph is said to be $k$-paintable if the remover has a winning strategy when $k$ tokens are available at each vertex. The paint number or online choice number of $G, \chi_{p}(G)$, is the smallest $k$ such that $G$ is $k$-paintable. We have that $\chi_{\ell}(G) \leqslant \chi_{p}(G)$, and there exist graphs where $\chi_{\ell}(G)<\chi_{p}(G)$ (see [5] and [19]).

Schauz [20] showed that if $D$ is an orientation of $G$ with maximum indegree $m$ such that $D$ satisfies the hypotheses of the Alon-Tarsi Theorem, then $\chi_{p}(G) \leqslant m+1$. So, whenever we use the Alon-Tarsi theorem to bound the list chromatic number of a graph, we actually get the same bound on the paint number of the graph which is a stronger result.

The Alon-Tarsi number of $G, A T(G)$, is the smallest $k$ for which there is an orientation, $D$, of $G$ with max indegree $k-1$ such that the number of even and odd circulations contained in $D$ are different (i.e. the smallest $k$ for which the hypotheses of the AlonTarsi Theorem are satisfied). Jensen and Toft [12] first defined and suggested this graph invariant for study. Hefetz [11] studied the AT-number and showed, among other results, that $A T(H) \leqslant A T(G)$ whenever $H$ is a subgraph of $G$. Recently, Zhu [24] showed that the AT-number of all planar graphs is at most 5, improving the classic list coloring bound on planar graphs.

In summary we know that:

$$
\chi(G) \leqslant \chi_{\ell}(G) \leqslant \chi_{p}(G) \leqslant A T(G) .
$$


In general all these inequalities can be strict, our aim is to find classes of graphs where they all are equal - a stronger form of chromatic-choosability. We study this phenomenon for Cartesian products of graphs.

\section{Applying the Alon-Tarsi Theorem}

In this section we will start by proving that the Cartesian product of an odd cycle and path is chromatic-choosable. Then, we prove several generalizations of this argument. Suppose that $G$ is the Cartesian product of an arbitrary odd cycle on $2 k+1$ vertices and an arbitrary path on $n$ vertices. Throughout the proof of Theorem 4 we assume that we form $G$ as follows. Suppose we place the vertices of $C_{2 k+1}$ around a circle so that two vertices are adjacent if and only if they appear consecutively along the circle, and we name the vertices in counterclockwise fashion around the circle as: $v_{1}, v_{2}, \ldots, v_{2 k+1}$ (we also call this ordering of the vertices cyclic order). Similarly we name the vertices of $P_{n}$ as: $w_{1}, w_{2}, \ldots, w_{n}$ so that two vertices are adjacent if and only if they appear consecutively in this list (we say that this ordering of the vertices is in order).

Theorem 4. For any $k, n \in \mathbb{N}, A T\left(C_{2 k+1} \square P_{n}\right)=3$. Consequently, $C_{2 k+1} \square P_{n}$ is chromaticchoosable.

Proof. The result is obvious when $n=1$. So, we assume that $n \geqslant 2$. Now, we orient the edges of $G=C_{2 k+1} \square P_{n}$ as follows. For each of the $n$ copies of $C_{2 k+1}$ in $G$ we orient the edges of each copy in counterclockwise fashion. Also we orient the edges of the form $\left\{\left(v_{i}, w_{j}\right),\left(v_{i}, w_{j+1}\right)\right\}$ so that $\left(v_{i}, w_{j}\right)$ is the tail and $\left(v_{i}, w_{j+1}\right)$ is the head (where $1 \leqslant i \leqslant 2 k+1$ and $1 \leqslant j \leqslant n-1)$. We call the oriented version of $G$ digraph $D$. We immediately note that for each $\left(v_{i}, w_{j}\right) \in V(D)$ :

$$
d_{D}^{-}\left(\left(v_{i}, w_{j}\right)\right)= \begin{cases}2 & \text { if } j \neq 1 \\ 1 & \text { if } j=1\end{cases}
$$

Now, let $G^{*}$ be the graph obtained from $G$ by adding an extra edge in $G$ with endpoints $\left(v_{1}, w_{1}\right)$ and $\left(v_{2}, w_{n}\right)$. Since $G$ is a subgraph of $G^{*}$, we have that $A T(G) \leqslant A T\left(G^{*}\right)$. We will now show that $A T\left(G^{*}\right) \leqslant 3$. To do this we form digraph $D^{*}$ by orienting the edges of $G^{*}$ so that all the edges of $G^{*}$ that are in $G$ are given the same orientation as in $D$ and the edge $\left\{\left(v_{1}, w_{1}\right),\left(v_{2}, w_{n}\right)\right\}$ is oriented so that $\left(v_{1}, w_{1}\right)$ is the head and $\left(v_{2}, w_{n}\right)$ is the tail. We call this oriented edge $e^{*}$. Note that for each $\left(v_{i}, w_{j}\right) \in V\left(D^{*}\right), d_{D^{*}}^{-}\left(\left(v_{i}, w_{j}\right)\right) \leqslant 2$.

To show the number of even and odd circulations contained in $D^{*}$ differ, we will prove that the number of circulations contained in $D^{*}$ is odd. We will refer to the $n$ oriented copies of $C_{2 k+1}$ in $D^{*}$ as the base cycles. We name the base cycles: $B_{1}, \ldots, B_{n}$ so that $B_{i}$ is the oriented copy of $C_{2 k+1}$ such that all the vertices in $B_{i}$ have second coordinate $w_{i}$. We note that the only directed cycles contained in $D^{*}$ are the base cycles and cycles that contain the edge $e^{*}$. Let $\mathcal{C}$ be the set of all circulations in $D^{*}$. We let

$$
\mathcal{A}=\left\{\begin{array}{l|l}
H \in \mathcal{C} & \begin{array}{l}
H \text { contains all the edges of at least one of the base cycles or } \\
H \text { does not include any edge from at least one of the base cycles }
\end{array}
\end{array}\right\} .
$$


Suppose $H \in \mathcal{A}$. We may assume $H$ contains all the edges from the base cycles: $B_{a_{1}}, \ldots, B_{a_{m}}$ and does not include any edges from the base cycles $B_{b_{1}}, \ldots, B_{b_{t}}$ (Note: at most one of these two lists of base cycles may be empty). Now, we form another circulation $H^{\prime} \in \mathcal{A}$ from $H$ as follows. We delete all the edges of the base cycles: $B_{a_{1}}, \ldots, B_{a_{m}}$ and we add all the edges of the base cycles: $B_{b_{1}}, \ldots, B_{b_{t}}$. We immediately note that $H \neq H^{\prime}$ and this mapping gives us a way to pair up distinct elements of $\mathcal{A}$. Thus, $|\mathcal{A}|$ is an even number.

Now, let $\mathcal{B}=\mathcal{C}-\mathcal{A}$. By the definition of $\mathcal{C}$ and $\mathcal{A}$ we have that $\mathcal{B}$ contains all the circulations of $D^{*}$ that contain at least one edge from each base cycle, but do not include all the edges of any base cycle. Suppose that $H \in \mathcal{B}$. We know that $H$ may be decomposed into directed cycles. No directed cycle in the decomposition of $H$ can be a base cycle. Since the only directed cycles in $D^{*}$ are the base cycles and cycles containing $e^{*}$, we may conclude that $H$ is a single cycle containing $e^{*}$ that contains at least one edge from each base cycle. This means that we can view each element of $\mathcal{B}$ as a directed cycle starting with vertex $\left(v_{1}, w_{1}\right)$ and ending with the edge $e^{*}$.

Now, for $q, r \in \mathbb{Z}, 0 \leqslant q \leqslant n-2$, and $0 \leqslant r \leqslant 2 k$, let $e_{(2 k+1) q+r}$ be the directed edge with tail $\left(v_{r+1}, w_{q+1}\right)$ and head $\left(v_{r+1}, w_{q+2}\right)$. For $0 \leqslant i \leqslant n-2$, we let:

$$
E_{i}=\left\{e_{(2 k+1) q+r} \mid q=i \text { and } 0 \leqslant r \leqslant 2 k\right\} .
$$

Intuitively, $E_{i}$ consists of the directed edges from the copies of $P_{n}$ that connect vertices in $B_{i+1}$ to vertices in $B_{i+2}$. Now, suppose that $\left(e_{a_{i}}\right)_{i=0}^{n-2}$ is a subsequence of the finite sequence of edges: $\left(e_{i}\right)_{i=0}^{(2 k+1)(n-1)-1}$. We call $\left(e_{a_{i}}\right)_{i=0}^{n-2}$ a level subsequence of edges if $e_{a_{i}} \in E_{i}$ for each $i, a_{1} \neq 0$, and $a_{i} \neq \equiv a_{i+1} \bmod 2 k+1$ for each $i \leqslant n-3$. Now, let $Q$ be the set of all level subsequences of edges, and let $R$ be the set of all level subsequences of edges that do not have the edge $e_{(2 k+1)(n-2)+1}$ as the last edge in the sequence.

We will now construct a bijection between $\mathcal{B}$ and $R$. Given a sequence of edges in $R$, $\left(e_{a_{i}}\right)_{i=0}^{n-2}$, there is a unique cycle in $D^{*}$ starting at $\left(v_{1}, w_{1}\right)$ and ending with the edge $e^{*}$ that includes each edge in $\left(e_{a_{i}}\right)_{i=0}^{n-2}$. To form this cycle, simply follow a portion of the base cycle $B_{1}$ to get from $\left(v_{1}, w_{1}\right)$ to $e_{a_{0}}$. The fact that $a_{1} \neq 0$ guarantees that we must traverse at least one edge of $B_{1}$. Then, for each $0 \leqslant i \leqslant n-3$, follow a portion of the base cycle $B_{i+2}$ to get from edge $e_{a_{i}}$ to $e_{a_{i+1}}$. The fact that $a_{i} \not \equiv a_{i+1} \bmod 2 k+1$ for each $i \leqslant n-3$ guarantees that we must traverse at least one edge of $B_{i+2}$. Finally, follow a portion of the base cycle $B_{n}$ to get from $e_{a_{n-2}}$ to $e^{*}$. The fact that $R$ contains all level subsequences that do not end with the edge incident to $e^{*}$ guarantees we must traverse at least one edge of $B_{n}$. We immediately notice from this construction that the cycle we form contains at least one edge from each base cycle, but does not include all the edges of any base cycle. Thus, the cycle we form is in $\mathcal{B}$. So, we have a function from $R$ to $\mathcal{B}$. To see that this function has an inverse suppose that $H \in \mathcal{B}$. We know that we can view $H$ as a directed cycle starting with $\left(v_{1}, w_{1}\right)$ and ending with the edge $e^{*}$. By the way in which $D^{*}$ is oriented we know that $H$ must alternate between edge(s) from $B_{i}$ and an edge from $E_{i-1}$ for $1 \leqslant i \leqslant n-1$. Then, cycle $H$ has edge(s) from $B_{n}$ followed by $e^{*}$. Let $\left(u_{k}\right)_{k=0}^{n-2}$ be the ordered sequence of edges in $H$ that are in $\cup_{i=0}^{n-2} E_{i}$. We immediately have that $u_{k} \in E_{k}$ for $0 \leqslant k \leqslant n-2$ by the way the paths are oriented. Since $H$ contains at least one edge 
from each base cycle, $u_{0} \neq e_{0}$, and if $u_{k}=e_{a^{\prime}}$ and $u_{k+1}=e_{b^{\prime}}$, then $a^{\prime} \not \equiv b^{\prime} \bmod 2 k+1$ for $0 \leqslant k \leqslant n-3$. So, $\left(u_{k}\right)_{k=0}^{n-2}$ is a level subsequence of edges by definition. Finally, since $H$ contains at least one edge from $B_{n}$, we know that $u_{n-2} \neq e_{(2 k+1)(n-2)+1}$. So, $\left(u_{k}\right)_{k=0}^{n-2} \in R$. We now have that our function maps $\left(u_{k}\right)_{k=0}^{n-2}$ to $H$ and the inverse maps $H$ to $\left(u_{k}\right)_{k=0}^{n-2}$. Thus, we have a bijection between $\mathcal{B}$ and $R$ and we conclude that $|\mathcal{B}|=|R|$.

Now, we will show that $|R|$ is an odd number. We note that $|Q|=(2 k)^{n-1}$ since forming an element of $Q$ leaves one with $2 k$ distinct choices at each step. We let $d_{r, n}$ be the number of elements in $Q$ that have a final edge with an index congruent to $r$ mod $2 k+1$ (Note: $n$ is the number of vertices in our path and $0 \leqslant r \leqslant 2 k$ ). The following recursive relationships are immediate:

$$
d_{r, n}=\sum_{i \in\{0, \ldots, 2 k\}, i \neq r} d_{i, n-1} \text { for } n \geqslant 3
$$

where $d_{0,2}=0$ and $d_{i, 2}=1$ for each $i \in\{1, \ldots 2 k\}$. An easy inductive argument shows that $d_{0, n}$ is even and $d_{i, n}$ is odd for each $i \in\{1, \ldots 2 k\}$ whenever $n \geqslant 2$. Since

$$
|R|=|Q|-d_{1, n}=(2 k)^{n-1}-d_{1, n},
$$

we may conclude that $|R|$ is an odd number which immediately implies $|\mathcal{B}|$ is an odd number.

Since $|\mathcal{C}|=|\mathcal{A}|+|\mathcal{B}|$, we have that $|\mathcal{C}|$ is odd. Thus, $D^{*}$ contains an odd number of circulations. This means that the number of even circulations in $D^{*}$ does not equal the number of odd circulations in $D^{*}$. So $A T\left(G^{*}\right) \leqslant 3$ and hence $A T(G) \leqslant 3$. We also have that $3 \leqslant \chi(G)$. So, $A T(G)=3$, and we are done.

Remark. It is easy to notice that in our proof of Theorem 4 we have proven something slightly stronger with regard to list coloring. Specifically we proved that if we have a list assignment, $L$, for $G$ that assigns two colors to all but one vertex in the first base cycle and three colors to all other vertices of $G$, then there is a proper $L$-coloring for $G$.

Having proven Theorem 4, it is easy to now classify the list chromatic number of the Cartesian product of an arbitrary cycle and path. Erdös et al. [6] classified all graphs with list chromatic number equal to 2 . Let $\Theta\left(l_{1}, \ldots, l_{k}\right)$ with branch vertices $u$ and $v$ be the graph that is the union of $k$ pairwise internally disjoint $u, v$-paths of lengths $l_{1}, \ldots, l_{k}$.

Theorem 5 ([6]). Let $G$ be a connected bipartite graph. Then, $\chi_{\ell}(G)=2$ if and only if $G$ has at most one cycle or the subgraph consisting of the non-cut-edges of $G$ is $\Theta(2,2,2 t)$ for some $t \in \mathbb{N}$.

This is the final ingredient in:

Corollary 6. For $k \in \mathbb{N}$ :

(i) $\chi\left(C_{2 k+1} \square P_{n}\right)=\chi_{\ell}\left(C_{2 k+1} \square P_{n}\right)=3$ for $n \in \mathbb{N}$,

(ii) $\chi\left(C_{2 k+2} \square P_{1}\right)=\chi_{\ell}\left(C_{2 k+2} \square P_{1}\right)=2$, and $\chi_{\ell}\left(C_{2 k+2} \square P_{n}\right)=3$ for $n \geqslant 2$. 
Proof. Statement (i) follows from Theorem 4. For statement (ii) notice that when $n \geqslant 2$, $C_{2 k+2} \square P_{n}$ contains more than one cycle and no cut edges. Moreover, all the vertices in $C_{2 k+2} \square P_{n}$ have degree at least 3 which means that $C_{2 k+2} \square P_{n}$ is not $\Theta(2,2,2 t)$ for any $t \in \mathbb{N}$. So, Theorem 5 implies that $3 \leqslant \chi_{\ell}\left(C_{2 k+2} \square P_{n}\right)$. By Theorem 5 , we also have that even cycles have list chromatic number equal to 2. So, Theorem 1 implies $\chi_{\ell}\left(C_{2 k+2} \square P_{n}\right) \leqslant 2+2-1=3$.

We now give a natural generalization of the argument of Theorem 4 .

Theorem 7. Suppose that $G$ is a complete graph or an odd cycle with $|V(G)| \geqslant 3$. Suppose $H$ is a graph on at least two vertices that contains a Hamilton path, $w_{1}, w_{2}, \ldots, w_{m}$, such that $w_{i}$ has at most $k$ neighbors among $w_{1}, \ldots, w_{i-1}$. Then, $A T(G \square H) \leqslant \Delta(G)+k$.

Before we prove this theorem a couple of remarks are worth making. We require $m \geqslant 2$, since we know $\chi_{\ell}(G \square H)=\Delta(G)+1$ when $m=1$. Now, suppose $G$ and $H$ satisfy the hypotheses of the Theorem. We have that $\chi_{\ell}(G)=\operatorname{col}(G)=\Delta(G)+1$ and $\operatorname{col}(H)-1 \leqslant k$. Theorem 1 tells us that $\chi_{\ell}(G \square H) \leqslant \Delta(G)+\chi_{\ell}(H)$, and Theorem 2 implies that $\chi_{\ell}(G \square H) \leqslant \Delta(G)+\Delta(H)$. So, Theorem 7 gives us an improvement on these known bounds if and only if $k<\chi_{\ell}(H)$ and $k<\Delta(H)$. It is easy to see that $k<\chi_{\ell}(H)$ and $k<\Delta(H)$ if and only if $k=\operatorname{col}(H)-1=\chi_{\ell}(H)-1$ and $d_{H}\left(w_{m}\right) \neq \Delta(H)$. We show examples in Section 3 where Theorem 7 improves these known bounds. We now present the proof.

Proof. Suppose $G$ has $n$ vertices. We name the vertices of $G$ (cyclically if $G$ is a cycle): $v_{1}, v_{2}, \ldots, v_{n}$. $G$ contains an odd cycle, $C$, as an induced subgraph. If $G$ is an odd cycle let $C=G$, and if $G$ is a complete graph let $C$ be the subgraph of $G$ induced by the vertices $v_{n-2}, v_{n-1}$, and $v_{n}$. For the remainder of the proof assume that $C$ has $2 t+1$ vertices where $t \in \mathbb{N}$ (Note: we know $t=1$ when $G$ is a complete graph). We also let $P$ be the Hamilton path, $w_{1}, w_{2}, \ldots, w_{m}$, contained in $H$.

Now, consider the graph $G \square H$. We form digraph $D$ from this graph by orienting its edges as follows. We begin by orienting each of the $m$ copies of $C$ in a counterclockwise fashion. Then, for each edge in a copy of $G$ and not in a copy of $C$ with endpoints $\left(v_{r}, w_{u}\right)$ and $\left(v_{s}, w_{u}\right)$ with $s>r$, we orient the edge so that $\left(v_{r}, w_{u}\right)$ is the tail and $\left(v_{s}, w_{u}\right)$ is the head. Finally, for each edge in a copy of $H$ with endpoints $\left(v_{u}, w_{r}\right)$ and $\left(v_{u}, w_{s}\right)$ with $s>r$, we orient the edge so that $\left(v_{u}, w_{r}\right)$ is the tail and $\left(v_{u}, w_{s}\right)$ is the head.

From $D$ we form the digraph $D^{*}$ by adding a directed edge with tail $\left(v_{n-2 t+1}, w_{m}\right)$ and head $\left(v_{n-2 t}, w_{1}\right)$. Note that $D^{*}$ is a simple digraph since $m \geqslant 2$. We will refer to the edge we added as $e^{*}$. We immediately note that by the conditions placed on $G$ and $H$, we have that $d_{D^{*}}^{-}\left(\left(v_{r}, w_{u}\right)\right) \leqslant \Delta(G)-1+k$ for each $\left(v_{r}, w_{u}\right) \in V\left(D^{*}\right)$. Similar to the proof of Theorem 4, we will now prove that the number of circulations in $D^{*}$ is odd. We will refer to the $m$ oriented copies of $C$ in $D^{*}$ as the base cycles. We name the base cycles: $B_{1}, \ldots, B_{m}$ so that $B_{i}$ is the oriented copy of $C$ such that all the vertices in $B_{i}$ have second coordinate $w_{i}$. We note that the only cycles in $D^{*}$ are the base cycles and the cycles that contain $e^{*}$. Let $S$ be the subgraph of $D^{*}$ that is made up of the $m$ oriented copies of $C$ in $D^{*}$, the oriented copies of $P$ that have first coordinate $v_{n-2 t}, v_{n-2 t+1}, \ldots$, 
and $v_{n}$, and the edge $e^{*}$. Notice that $S$ is $C \square P$ plus edge $e^{*}$ oriented as it is in the proof of Theorem 4. Let $\mathcal{C}$ be the set of all circulations in $D^{*}$. We define $\mathcal{A}$ as in the proof of Theorem 4. By the same argument as in the proof of Theorem 4 , we see that $|\mathcal{A}|$ is even. Now, let $\mathcal{B}=\mathcal{C}-\mathcal{A}$. As in the proof of Theorem 4 , we have that any $K \in \mathcal{B}$ is a single cycle containing $e^{*}$ that contains at least one edge from each base cycle. Moreover, any $K \in \mathcal{B}$ must be completely contained in $S$ (since any vertex of the form $\left(v_{\ell}, w_{u}\right)$ with $l<n-2 t$ is in no cycles of $D^{*}$ and any cycle in $D^{*}$ that contains an oriented edge of a copy of $H$ that is not in a copy of $P$ must leave out at least one base cycle).

Thus, by the same argument as in the proof of Theorem 4 , we have that $|\mathcal{B}|$ is odd. Thus, $|\mathcal{C}|$ is odd, and we have the desired result by the Alon-Tarsi Theorem.

Remark. Note that our proof also works when $G$ is any graph that contains an induced odd cycle $C$ such that every maximum degree vertex in $G$ is either in $C$ or adjacent to $C$. However, this is not particularly useful for bounding $\chi_{\ell}(G \square H)$, since when $G$ is not an odd cycle or complete graph Theorems 2 and 1 yield:

$$
\chi_{\ell}(G \square H) \leqslant \chi_{\ell}(G)+\operatorname{col}(H)-1 \leqslant \Delta(G)+k+1-1=\Delta(G)+k .
$$

Now, we present another extension of Theorem 4. We first need some definitions and notation. Suppose that $G$ is an arbitrary graph, and $T$ is some subset of $V(G)$. We write $G[T]$ for the subgraph of $G$ induced by the vertices in $T$. Now, suppose that $G_{1}$ and $G_{2}$ are two arbitrary vertex disjoint graphs. The join of the graphs $G_{1}$ and $G_{2}$, denoted $G_{1} \vee G_{2}$, is the graph consisting of $G_{1}, G_{2}$, and additional edges added so that each vertex in $G_{1}$ is adjacent to each vertex in $G_{2}$. When a graph, $G$, consists of $G_{1}, G_{2}$, and some set of additional edges (possibly empty) that have one endpoint in $V\left(G_{1}\right)$ and the other endpoint in $V\left(G_{2}\right)$ we say that $G$ is a partial join of $G_{1}$ and $G_{2}$.

Theorem 8. Suppose that $G$ is a graph with the property that there exists a partition, $\left\{S_{1}, \ldots, S_{m}\right\}$, of $V(G)$ such that for each $i G\left[S_{i}\right]$ is an odd cycle. For each $i \geqslant 2$ suppose each vertex in $S_{i}$ has at most $\rho$ neighbors in $\cup_{j=1}^{i-1} S_{j}$ (we let $\rho=0$ in the case that $m=1$ ). Then, $A T\left(G \square P_{k}\right) \leqslant 3+\rho$, for any $k \in \mathbb{N}$.

Note that we obtain Theorem 4 when $m=1$. We also notice that when $m=2, G$ is a partial join of two odd cycles. When $m=3, G$ is a partial join of the odd cycle $G\left[S_{3}\right]$ and $G\left[S_{1} \cup S_{2}\right]$ (where $G\left[S_{1} \cup S_{2}\right]$ is itself the partial join of two odd cycles). And so on.

Proof. First, for each $i$, we index the vertices of $G\left[S_{i}\right]$ in cyclic order as: $v_{i, 1}, v_{i, 2}, \ldots, v_{i, m_{i}}$ where we know that $m_{i}$ is odd for each $i$. If $e \in E(G)$ is an edge with one endpoint in $S_{i}$ and one endpoint in $S_{j}$ with $i \neq j$ we refer to $e$ as a connecting edge.

We now turn our attention to the case where $k=1$. In this case, we must show that $A T(G) \leqslant 3+\rho$. We form an oriented version, $D$, of the graph $G$ as follows. For each odd cycle, $G\left[S_{i}\right]$, orient the edges of the cycle so that $v_{i, l}$ is the tail and $v_{i, l+1}$ is the head for $1 \leqslant l \leqslant m_{i}-1$. Then, orient the final edge of the cycle so that $v_{i, 1}$ is the tail and $v_{i, m_{i}}$ is the head. Finally, for each connecting edge, $e \in E(G)$, with one endpoint in $S_{i}$ and one 
endpoint in $S_{j}$ with $i<j$ orient $e$ so that its tail is in $S_{i}$ and its head is in $S_{j}$. One may now note that for each $v \in S_{i}$,

$$
d_{D}^{-}(v) \leqslant 2+\rho
$$

We now claim that $D$ is acyclic. First, note that no oriented connecting edge can be in a cycle in $D$ since there is no way in $D$ to get from a vertex in $S_{j}$ to a vertex in $S_{i}$ when $i<j$. This means that if there is a cycle, $C$, in $D$ there must exist an $i$ such that the vertices of $C$ are a subset of the vertices of $S_{i}$. However, $D\left[S_{i}\right]$ is acyclic by construction. So, no such $C$ can exist. This means that $D$ has one even circulation (the circulation with no edges) and zero odd circulations. Then $A T(G) \leqslant 3+\rho$ as desired.

We now turn our attention to the case where $k \geqslant 2$. Let $H=P_{k}$. We note that $G \square H$ is made of the disjoint union of graphs:

$$
\sum_{i=1}^{m}\left(G\left[S_{i}\right] \square H\right)
$$

plus the connecting edges in each of the $k$ copies of $G$. Let $G^{* *}$ be the graph formed from $G \square H$ by adding an edge in $G\left[S_{i}\right] \square H$ for each $i$ as we did in the proof of Theorem 4 to form $G^{*}$. We call the newly formed subgraph of $G^{* *}$ consisting of $G\left[S_{i}\right] \square H$ plus an additional edge, $M_{i}$ for each $i$. We form an oriented version, $D$, of $G^{* *}$ as follows. For each subgraph $M_{i}$ of $G^{* *}$, we orient this subgraph just as we oriented $D^{*}$ in the proof of Theorem 4. Finally, we orient all the connecting edges in all the copies of $G$ just as we did in the case of $k=1$. One may note that for each $v \in V(D)$, we have that $d_{D}^{-}(v) \leqslant 2+\rho$. Similar to the case where $k=1$, we note that no connecting edge from any copy of $G$ is in a cycle contained in $D$. This means that if $C_{i}$ is the number of circulations in the oriented version of $M_{i}$, the number of circulations in $D$ is equal to

$$
\prod_{i=1}^{m} C_{i}
$$

which by the proof of Theorem 4 is an odd number. Thus, the number of even circulations in $D$ does not equal the number of odd circulations in $D$, and we have that $A T(G \square H) \leqslant$ $A T\left(G^{* *}\right) \leqslant \rho+3$.

Finally, we can easily combine the idea of the proofs of Theorems 7 and 8 to obtain the following.

Theorem 9. Suppose that $G$ is a graph with the property that there exists a partition, $\left\{S_{1}, \ldots, S_{m}\right\}$, of $V(G)$ such that for each $i\left|S_{i}\right| \geqslant 3$ and $G\left[S_{i}\right]$ is an odd cycle or a complete graph. For each $i \geqslant 2$ suppose each vertex in $S_{i}$ has at most $\rho_{i}$ neighbors in $\cup_{j=1}^{i-1} S_{j}$, and let $\rho_{1}=0$. For each $i \geqslant 1$ we let:

$$
\alpha_{i}= \begin{cases}\rho_{i}+3 & \text { if } G\left[S_{i}\right] \text { is an odd cycle } \\ \rho_{i}+\left|S_{i}\right| & \text { if } G\left[S_{i}\right] \text { is a complete graph } .\end{cases}
$$


Now, let $\alpha=\max _{i} \alpha_{i}$. Suppose $H$ is a graph on at least two vertices that contains a Hamilton path, $w_{1}, w_{2}, \ldots, w_{n}$, such that $w_{i}$ has at most $k$ neighbors among $w_{1}, \ldots, w_{i-1}$. Then, $A T(G) \leqslant \alpha$ and $A T(G \square H) \leqslant \alpha+k-1$.

Both Theorems 8 and 9 are sharp and give improvements over existing bounds as shown in examples in the next section.

\section{Some Examples}

In this section we present some examples where our results from Section 2 improve upon known bounds for the list chromatic number. We let the kth power of graph $G$, denoted $G^{k}$, be the graph with vertex set $V(G)$ where two vertices are adjacent if their distance in $G$ is at most $k$. It is easy to see that $P_{n}^{r}, 1 \leqslant r \leqslant n-1$, satisfies $\chi\left(P_{n}^{r}\right)=\chi_{\ell}\left(P_{n}^{r}\right)=$ $\operatorname{col}\left(P_{n}^{r}\right)=r+1$. We already know Theorem 7 is sharp by $r=1$ below.

Corollary 10. Suppose that $k, r, n \in \mathbb{N}$ are such that $n \geqslant 2$ and $r \leqslant n-1$.

Then, $\max \{r+1,3\} \leqslant A T\left(C_{2 k+1} \square P_{n}^{r}\right) \leqslant r+2$.

Proof. Let $G=C_{2 k+1}$ and $H=P_{n}^{r}$. Name the vertices of $H: w_{1}, w_{2}, \ldots, w_{n}$ so that $w_{i}$ is the $i^{t h}$ vertex of the underlying path on $n$ vertices contained in $H$. Clearly, $H$ contains the Hamilton path: $w_{1}, w_{2}, \ldots, w_{n}$, and for each $i, w_{i}$ has at most $r$ neighbors among $w_{1}, w_{2}, \ldots, w_{i-1}$. Thus, by Theorem 7 , we have that $A T(G \square H) \leqslant 2+r$.

We note that Theorem 1 only yields that $\chi_{\ell}(G \square H) \leqslant r+3$. Also, Theorem 2 yields $\chi_{\ell}(G \square H) \leqslant 2+\Delta\left(P_{n}^{r}\right)$, and $r+1 \leqslant \Delta\left(P_{n}^{r}\right)$ when $n \geqslant 3$ and $r \leqslant n-2$. So, the above example improves upon known bounds on the list chromatic number when $n \geqslant 3$ and $r \leqslant n-2$. We suspect that $C_{2 k+1} \square P_{n}^{r}$ is often chromatic-choosable, but improving upon our upper bound with the Alon-Tarsi Theorem seems difficult. For example, it would be impossible to find an orientation of $C_{2 k+1} \square P_{n}^{2}$ with max indegree of 2 for large values of $n$.

Before we move on to an example for Theorems 8 and 9, let us note a couple of other facts implied by Theorem 7: $A T\left(K_{n} \square P_{m}\right)=n$, for $n \geqslant 3$; and $n-2 \leqslant A T\left(C_{2 k+1} \square H_{n}\right) \leqslant$ $n-1$ where $H_{n}=K_{n}-E\left(P_{4}\right)$ for $n \geqslant 5$.

Corollary 11. For $G=K_{m} \vee C_{2 k+1}, A T\left(G \square P_{n}\right)=m+3$, where $m, k, n \in \mathbb{N}$. Consequently, $\left(K_{m} \vee C_{2 k+1}\right) \square P_{n}$ is chromatic-choosable.

Proof. The result is obvious when $n=1$ since $\operatorname{col}\left(K_{m} \vee C_{2 k+1}\right)=m+3$. So, suppose $n \geqslant 2$. Let $G_{1}=K_{m}$ and $G_{2}=C_{2 k+1}$. Since $\chi\left(G_{1} \vee G_{2}\right)=\chi\left(G_{1}\right)+\chi\left(G_{2}\right)$, we have that $m+3=\chi(G)=\chi\left(G \square P_{n}\right)$.

First, consider the case where $m \geqslant 3$. Let $\left\{S_{1}, S_{2}\right\}$ be the partition of $V(G)$ where $S_{1}=V\left(G_{1}\right)$ and $S_{2}=V\left(G_{2}\right)$. Note $G\left[S_{1}\right]$ is a complete graph, and $G\left[S_{2}\right]$ is an odd cycle. Also, each vertex in $S_{2}$ has exactly $m$ neighbors in $S_{1}$. So, by Theorem 9 we have that $A T\left(G \square P_{n}\right) \leqslant m+3$.

Finally, suppose $m=1,2$. Let $G^{(m)}$ be a partial join of $G_{3}=C_{3}$ and $G_{2}$ so that $m$ vertices in $V\left(G_{3}\right)$ are adjacent to all the vertices in $V\left(G_{2}\right)$, and the other $3-m$ vertices 
in $V\left(G_{3}\right)$ are not adjacent to any of the vertices in $V\left(G_{2}\right)$. Now, let $\left\{S_{3}, S_{4}\right\}$ be the partition of $V\left(G^{(m)}\right)$ where $S_{3}=V\left(G_{3}\right)$ and $S_{4}=V\left(G_{2}\right)$. Note $G^{(m)}\left[S_{3}\right]$ and $G^{(m)}\left[S_{4}\right]$ are odd cycles, and each vertex in $S_{4}$ has exactly $m$ neighbors in $S_{3}$. So, by Theorem 8 we have that $A T\left(G^{(m)} \square P_{n}\right) \leqslant m+3$. The result follows since $G \square P_{n}$ is a subgraph of $G^{(m)} \square P_{n}$.

We note that when $n \geqslant 2$ Theorem 1 only yields that $\chi_{\ell}\left(\left(K_{m} \vee C_{2 k+1}\right) \square P_{n}\right) \leqslant m+4$. Also, when $n \geqslant 3$, Theorem 2 only tells us $\chi_{\ell}\left(\left(K_{m} \vee C_{2 k+1}\right) \square P_{n}\right) \leqslant \max \{m+4, m+2 k+2\}$.

There is still much to be discovered about the list chromatic number of the Cartesian product of graphs. Aside from the important conjectures proposed in [4], this paper also provides us with some interesting questions. Specifically, an ambitious question would be: Can we determine when $G \square H$ will be chromatic-choosable based upon some property of the factors? We further explore this question in a following paper [14]. Simpler questions, motivated by Corollaries 6 and 10, include: For what graphs $G$, is $G \square P_{n}$ chromaticchoosable? When is $C_{2 k+1} \square P_{n}^{r}$ chromatic-choosable? For the first of these questions one may conjecture based upon the results of this paper that if $G$ is chromatic-choosable and $\chi(G) \geqslant 3$, then $G \square P_{n}$ is chromatic-choosable. However, this conjecture is false since one can construct a 3 -assignment to show that $3<\chi_{\ell}\left(C_{6}^{2} \square P_{2}\right)$ (see [15] for further details).

\section{References}

[1] N. Alon, Degrees and choice numbers, Random Structures Algorithms 16 (2000), 364-368.

[2] N. Alon and M. Tarsi, Colorings and orientations of graphs, Combinatorica 12 (1992), 125-134.

[3] O. V. Borodin, A. V. Kostochka, and D. R. Woodall, List edge and list total colourings of multigraphs, J. Combin. Theory Ser. B 71 (1997), no. 2, 184-204.

[4] M. Borowiecki, S. Jendrol, D. Král, and Miškuf, List coloring of cartesian products of graphs, Discrete Mathematics 306 (2006), 1955-1958.

[5] J. Carraher, S. Loeb, T. Mahoney, G. Puleo, M. Tsai, and D. B. West, Three topics in online list coloring, Journal of Combinatorics 5 (2014), no. 1, 115-130.

[6] P. Erdős, A. L. Rubin, and H. Taylor, Choosability in graphs, Congressus Numerantium 26 (1979), 125-127.

[7] F. Galvin, The list chromatic index of a bipartite multigraph, J. Combinatorial Theory Series B 63 (1995), no. 1, 153-158.

[8] S. Gravier, and F. Maffray, Choice number of 3-colorable elementary graphs, Discrete Math. 165/166 (1997), 353-358, Graphs and combinatorics (Marseille, 1995).

[9] R. Häggkvist and A. Chetwynd, Some upper bounds on the total and list chromatic numbers of multigraphs, J. Graph Theory 16 (1992), no. 5, 503-516.

[10] Hajós, G., Über eine Konstruktion nicht $n$-färbbarer graphen, Wiss. Z. MartinLuther-Univ. Halle-Wittenberg Math.-Natur. Reihe 10 (1961), 116-117. 
[11] D. Hefetz, On two generalizations of the Alon-Tarsi polynomial method, J. Combin. Theory Ser. B 101 (2011), no. 6, 403-414.

[12] T. Jensen, B. Toft, Graph Coloring Problems, Wiley, New York, 1995.

[13] J. Kahn, Asymptotically good list-colorings, J. Combin. Theory Ser. A 73 (1996), no. $1,1-59$.

[14] H. Kaul, J. Mudrock, Criticality, List Color Function, and List Coloring the Cartesian Product of Graphs, arXiv: 1805.02147, 2018.

[15] J. Mudrock, On the list coloring problem and its equitable variants, Ph.D. Thesis, Illinois Institute of Technology, 2018.

[16] J. A. Noel, B. A. Reed, H. Wu, A proof of a conjecture of Ohba, J. Graph Theory 79 (2) (2015), 86-102.

[17] K. Ohba, On chromatic-choosable graphs, J. Graph Theory 40 (2002), no. 2, 130-135.

[18] A. Prowse and D.R. Woodall, Choosability of powers of circuits, Graphs Combin. 19 (2003), 137-144.

[19] U. Schauz, Mr. Paint and Mrs. Correct, The Electronic Journal of Combinatorics 16(1) (2009), \#R77.

[20] U. Schauz, Flexible lists in Alon and Tarsi's theorem, and time scheduling with unreliable participants, The Electronic Journal of Combinatorics 17(1) (2010), \#R13.

[21] Zs. Tuza and M. Voigt, On a conjecture of Erdős, Rubin, and Taylor, Tatra Mt. Math Publ. 9 (1996), 69-82.

[22] V. G. Vizing, Coloring the vertices of a graph in prescribed colors, Diskret. Analiz. no. 29, Metody Diskret. Anal. v Teorii Kodovi Skhem 101(1976), 3-10.

[23] D. B. West, (2001) Introduction to Graph Theory. Upper Saddle River, NJ: Prentice Hall.

[24] X. Zhu, The Alon-Tarsi number of planar graphs, J. Combinatorial Theory, Series B, 134, (2019), 354-358. 